\title{
A shooting argument approach to a sharp-type solution for nonlinear degenerate Fisher-KPP equations
}

\author{
Faustino Sánchez-Garduñoł and Philip K. Mainl \\ Centre for Mathematical Biology, Mathematical Institute, University of Oxford, \\ 24-29 St Giles', Oxford OXI 3LB, UK
}

M. E. KAPPOS

Department of Applied Mathematics, University of Sheffield, Sheffield S10 2UN, UK

[Received 25 August 1995]

\begin{abstract}
In this paper we prove the existence and uniqueness of a travelling-wave solution of sharp type for the degenerate (at $u=0$ ) parabolic equation $u_{t}=\left[D(u) u_{x}\right]_{x}+$ $g(u)$ where $D$ is a strictly increasing function and $g$ is a function which generalizes the kinetic part of the classical Fisher-KPP equation. The original problem is transformed into the proper travelling-wave variables, and then a shooting argument is used to show the existence of a saddle-saddle heteroclinic trajectory for a critical value, $c^{*}>0$, of the speed $c$ of an autonomous system of ordinary differential equations. Associated with this connection is a sharp-type solution of the nonlinear partial differential equation.
\end{abstract}

\section{Introduction}

A wide range of wave behaviour has been observed in biological, ecological, physiological, and chemical systems. For example, spiral waves of concentration with one or multiple arms in chemical reactions, or of the density in the streaming behaviour of the amoeba Dictyostelium discoideum, scroll-like patterns in myocardium muscle, pulses of voltage in nerve conduction, waves of invasion of biological populations, and epizootic waves of disease spreading (see $[5,10,17$, $18,25,26]$ and the references therein). Some of these phenomena can be described by nonlinear reaction-diffusion equations (see $[5,9,17]$, for example). In many such cases, the observed waves can be approximated by waves moving in space with a constant profile and speed. These are the so-called travelling waves. Different mathematical methods have been developed and used in analysing the existence of travelling-wave solutions of systems of reaction-diffusion equations in one and higher dimensions (see $[2,6,12,14,15,16,21,23,24]$, for example). The classical example is due to Kolmogorov et al. (see [15]) in which the problem is restated in terms of finding the set of parameters (including the speed $c$ ) for which heteroclinic and homoclinic trajectories exist of an autonomous ordinarydifferential-equation (ODE) system in the travelling-wave variable.

†Departamento de Mathemáticas, Facultad de Ciencias, UNAM, Circuito Exterior, $C U$, México $04510, D F$, Mexico. 
Several authors (see $[1,8,12,13,15])$ have used the approach above to prove the existence of different types of travelling-wave solutions for some nonlinear reaction-diffusion equations, such as the classical Fisher-KPP and Nagumo equations given by $u_{t}=\left[D u_{x}\right]_{x}+g(u)$, where $D$ is a positive constant and $g$ is a second- or third-order polynomial. For a strictly positive density-dependent diffusion coefficient $D$, and when $g$ is typically a second-order polynomial-like function, the problem of the existence of travelling-wave solutions is wholly solved $[7,13]$. These authors proved that this equation has travelling-wave solutions if and only if the equation $u_{t}=u_{x x}+D(u) g(u)$ exhibits travelling-wave solutions. By using the aforementioned methodology it has been proved [23] that under certain conditions on $D$ and $g$ (see Section 2) the degenerate (at $u=0$ ) equation $u_{t}=\left[D(u) u_{x}\right]_{x}+g(u)$ has: (i) a unique sharp travelling-wave solution for a critical value, $c^{*}>0$, of $c$; (ii) no travelling-wave solution for $0<c<c^{*}$; and (iii) a travelling-wave solution of monotonic decreasing front type for each $c>c^{*}$. The existence and uniqueness of a sharp-type solution for the equation with $D(u)=u$ and with $g$ quadratic-like has been proved in [11].

Here we prove a result on the existence and uniqueness of a travelling-wave solution of the sharp type for equation (1); a different, less direct, proof can be found in [23]. The paper is organized as follows: In Section 2 we state the problem and address some background results on the nonlinear local analysis and global behaviour of the trajectories of the ODE system for certain values of $c$. Section 3 deals with the proof of the existence of the saddle-saddle heteroclinic trajectory. In Section 4 we present a result on the uniqueness of the saddlesaddle connection. Corresponding to this trajectory is the sharp solution of the full partial differential equation (PDE).

\section{The problem and some previous results}

The problem we consider is that of looking for a travelling-wave solution $u(x, t)=\phi(x-c t) \equiv \phi(\xi)$ of sharp type (see Definition 1) to the nonlinear, degenerate (at $u=0$ ), one-dimensional, reaction-diffusion equation

$$
\frac{\partial u}{\partial t}=\frac{\partial}{\partial x}\left[D(u) \frac{\partial u}{\partial x}\right]+g(u) \quad \forall(x, t) \in \mathbb{R} \times \mathbb{R}^{+},
$$

where $D$ and $g$ are functions defined on $[0,1]$ satisfying the following conditions:

(i) $g(0)=g(1)=0, \quad g(u)>0 \quad \forall u \in(0,1)$,

(ii) $g \in \mathrm{C}_{[0,1]}^{2}, \quad g^{\prime}(0)>0, \quad g^{\prime}(1)<0$,

(iii) $D(0)=0, \quad D(u)>0 \quad \forall u \in(0,1]$,

(iv) $D \in \mathrm{C}_{[0,1]}^{2}, \quad D^{\prime}(u)>0 \quad \forall u \in(0,1]$, with $D^{\prime \prime}(0) \neq 0$,

together with the initial condition $u(x, 0)=u_{0}(x)$ such that $0 \leqslant u_{0}(x) \leqslant 1$. Also we require $0 \leqslant \phi(\xi) \leqslant 1 \quad \forall \xi$.

For the biological and ecological motivation for analysing the above equation, see $[3,4,18,19,23]$. Note that, because of condition (i), $u_{1}(x, t) \equiv 0$ and 
$u_{2}(x, t) \equiv 1$ are homogeneous and stationary solutions of $(1)$. Thus it is natural to consider those travelling-wave solutions of (1) which connect these two equilibrium states.

Before we begin the analysis we introduce the following definition.

DEFINITION 1 If there exists a value $c^{*}>0$ of the speed $c$ and a value $\xi^{*} \in$ $(-\infty,+\infty)$ of $\xi$ such that $u(x, t)=\phi\left(x-c^{*} t\right)=\phi(\xi)$ satisfies

$$
\text { 1. } D(\phi) \phi^{\prime \prime}+c^{*} \phi^{\prime}+D^{\prime}(\phi)[\phi]^{2}+g(\phi)=0 \quad \forall \xi \in\left(-\infty, \xi^{*}\right) \text {, }
$$

2. $\phi(-\infty)=0, \quad \phi\left(\xi^{*-}\right)=\phi\left(\xi^{*^{+}}\right)=0, \quad$ and $\phi(\xi)=0 \quad \forall \xi \in\left(\xi^{*},+\infty\right]$,

3. $\phi^{\prime}\left(\xi^{*-}\right)=-\frac{c^{*}}{D^{\prime}(0)}, \quad \phi^{\prime}\left(\xi^{*+}\right)=0$, and $\phi^{\prime}(\xi)<0 \quad \forall \xi \in\left(-\infty, \xi^{*}\right)$,

then the function $u(x, t)=\phi\left(x-c^{*} t\right)$ is called a travelling-wave solution of sharp type for equation (1).

By substituting $u(x, t)=\phi(\xi)$ into (1) we obtain a second-order ODE of the form given by (2), which can be written, on introducing $v=\phi^{\prime}(\xi)$, as the singular (at $\phi=0$ ) system

$$
\begin{aligned}
\phi^{\prime} & =v, \\
D(\phi) v^{\prime} & =-c v-D^{\prime}(\phi) v^{2}-g(\phi),
\end{aligned}
$$

where the primes on $\phi$ and $v$ denote the derivative with respect to $\xi$ and the prime on $D$ denotes the derivative with respect to $\phi$. The singularity can be removed by using a standard reparametrization (see $[3,23]$ ) of $(3)$. Thus, letting $\tau$ be such that

$$
\frac{\mathrm{d} \tau}{\mathrm{d} \xi}=\frac{1}{D(\phi(\xi))}
$$

and defining $\phi(\tau) \equiv \phi(\xi(\tau))$ and $v(\tau) \equiv v(\xi(\tau))$, we can rewrite (3) as the nonsingular system

$$
\begin{aligned}
& \dot{\phi}=D(\phi) v, \\
& \dot{v}=-c v-D^{\prime}(\phi) v^{2}-g(\phi),
\end{aligned}
$$

where the overdots on $\phi$ and $v$ denote the derivative with respect to $\tau$. This system, together with the boundary conditions $\phi(-\infty)=1, v(-\infty)=0$, and $\phi(+\infty)=0$, with $0 \leqslant \phi(\tau) \leqslant 1$ and $v(\tau) \leqslant 0 \forall \tau \in(-\infty,+\infty)$, constitutes the restatement of problem (1).

It can be proved that systems (3) and (5) give the same dynamics in the strip $\{(\phi, v): 0<\phi \leqslant 1,-\infty<v<+\infty\}$; that is, they are topologically equivalent in this region of the $(\phi, v)$-plane. 


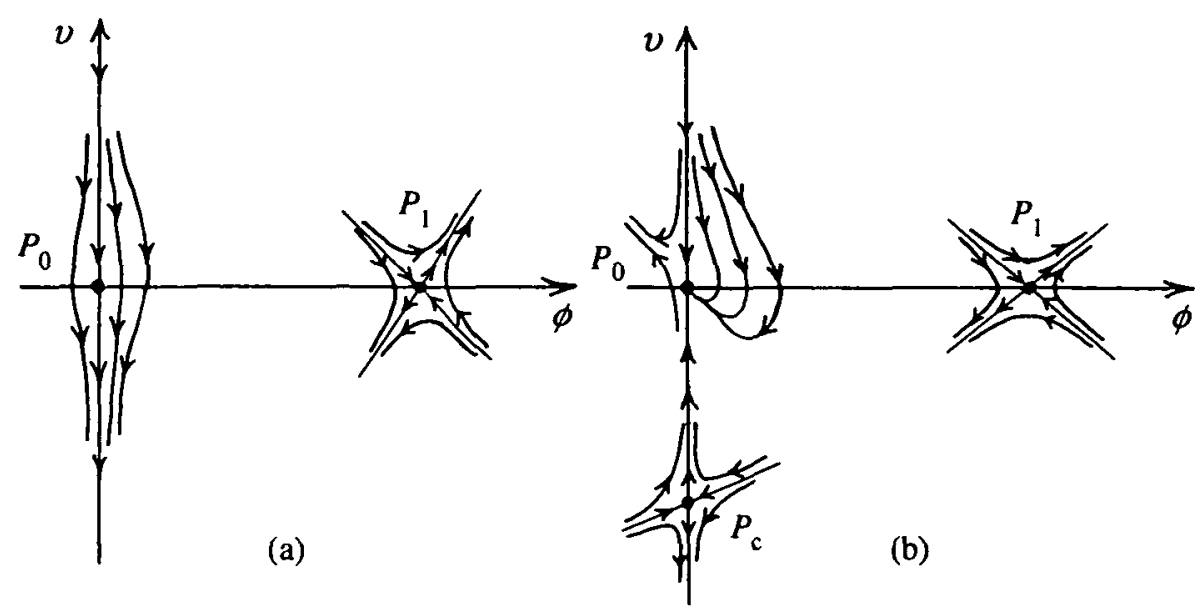

Flg. 1. The local behaviour of the trajectories of (5) around each equilibrium point for different values of $c$ : (a) $c=0$, and (b) $c>0$.

We begin the analysis by considering the local behaviour. System (5) has three equilibria: $P_{0}=(0,0), P_{1}=(1,0)$, and $P_{c}=\left(0,-c / D^{\prime}(0)\right) ; P_{c}$ and $P_{1}$ are hyperbolic saddle points for all positive values of $c ; P_{0}$ is a nonhyperbolic saddle-node point which runs away monotonically on the negative $v$-axis as $c$ increases. The local phase portrait of (5) is illustrated in Fig. 1. For full details of the nonlinear local analysis, see [23].

For the global phase portrait of (5) we introduce the following notation: $W_{c}^{u}\left(P_{1}\right)$ is the left unstable manifold of $P_{1}$, and $W_{c}^{s}\left(P_{c}\right)$ is the right stable manifold of $P_{c}$. We also define the region $\mathscr{R} \equiv\{(\phi, v): 0 \leqslant \phi \leqslant 1,-\infty<v \leqslant 0\}$. We need the following results which give us the global behaviour of $W_{c}^{u}\left(P_{1}\right)$ and $W_{c}^{s}\left(P_{c}\right)$ for some values of $c$. The proof can be seen in [23].

Proposition 1 For sufficiently small positive values of $c$, the path, $v_{c}(\phi)$, of $W_{c}^{u}\left(P_{1}\right)$ tends to $-\infty$ as $\phi \rightarrow 0^{+}$while $W_{c}^{s}\left(P_{c}\right)$ leaves (in reverse time) the region $\mathscr{R}$ at some point $\phi_{0}$ situated to the right of $P_{0}$ but sufficiently close to $P_{0}$. The phase portrait of (5) is illustrated in Fig. 2(a).

We set $M \equiv \max \left[4 D^{\prime}(\phi) g(\phi)\right]$, where the maximum is taken on the interval $[0,1]$. The following lemma holds:

LEMMA 1 For each $c>0$ such that $c^{2} \geqslant M$ there exists a heteroclinic trajectory of (5) connecting $P_{1}$ with $P_{0}$. Moreover, the trajectory $W_{c}^{s}\left(P_{c}\right)$ leaves (in reverse time) the region $\mathscr{R}$ somewhere on the semi-infinite interval $-\infty<v<0$ with $\phi \equiv 1$. The phase portrait of (5) is illustrated in Fig. 2(b).

REMARK 1 Given the equivalence between looking for travelling-wave solutions for (1) satisfying appropriate boundary conditions and looking for heteroclinic trajectories for system (5), the meaning of Proposition 1 and Lemma 1 is as follows: (i) for sufficiently small positive values of $c$ there are no travelling-wave solutions for (1); (ii) for each $c>0$, such that $c^{2} \geqslant M$, there exists a monotone 


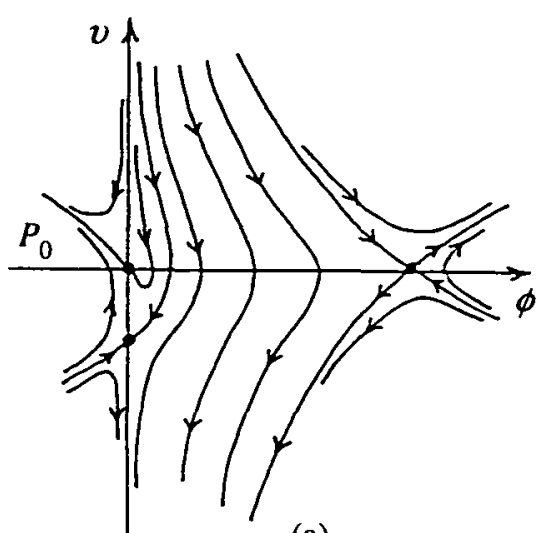

(a)

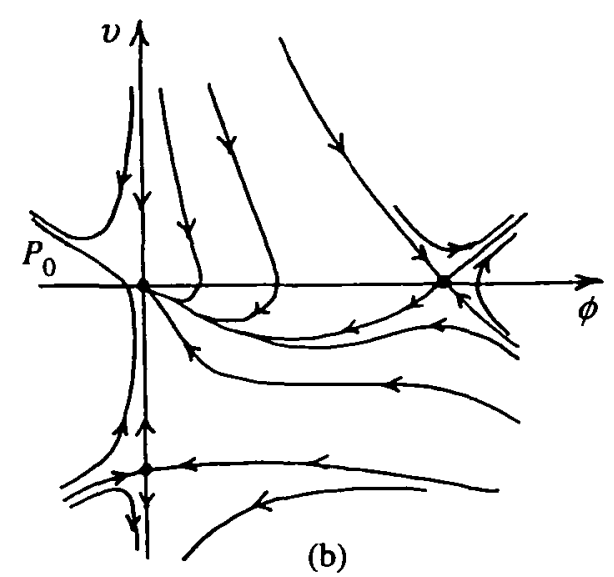

(b)

FIG. 2. The phase portrait of (5) for extremum values of $c$. (a) For sufficiently small positive values there are no connections; that is, there are no travelling-wave solutions. (b) For each $c>0$ such that $c^{2} \geqslant M$, for which there exists a saddle-saddle-node heteroclinic trajectory which corresponds to a travelling-wave solution for (1) of the front type.

decreasing travelling-wave solution of the front type for equation (1) satisfying the boundary conditions, $\phi(-\infty)=1, \dot{\phi}(-\infty)=0$, and $\phi(+\infty)=0, \dot{\phi}(+\infty)=0$, with $0 \leqslant \phi(\tau) \leqslant 1$ for all $\tau$.

Hereafter we let $c_{0}$ denote a sufficiently small positive value of $c$ and we set $I_{M} \equiv\left[c_{0}, M^{\frac{1}{2}}\right]$. Let $c_{1}$ and $c_{2}$ be two arbitrary values of the speed $c$ on $I_{M}$, with $c_{1}<c_{2}$. Define $v_{c_{1}}(\phi)$ and $v_{c_{2}}(\phi)$ as the path of $W_{c_{1}}^{s}\left(P_{c_{1}}\right)$ and $W_{c_{2}}^{s}\left(P_{c_{2}}\right)$, respectively, whose graphs belong to the region $\mathscr{R}$. In a similar way we define $v_{c_{0}}(\phi)$ and $v_{M^{1}}(\phi)$. The following proposition can be proved (see [23]):

Proposition 2 For $c_{0}<c_{1}<c_{2}<M^{\frac{1}{2}}$ the following inequalities hold

$$
v_{M^{\frac{1}{2}}}(\phi)<v_{c_{2}}(\phi)<v_{c_{1}}(\phi)<v_{c_{0}}(\phi)
$$

for all $\phi \in[0,1]$.

Remark 2 By Proposition 2, if $W_{c_{1}}^{s}\left(P_{c_{1}}\right)$ and $W_{c_{2}}^{s}\left(P_{c_{2}}\right)$ leave $\mathscr{R}$ somewhere on $(0,1)$, then $W_{c_{2}}^{s}\left(P_{c_{2}}\right)$ does so to the right of $W_{c_{1}}^{s}\left(P_{c_{1}}\right)$. If both leave $\mathscr{R}$ on $\{(\phi, v): \phi \equiv 1, v<0\}$, then $W_{c_{2}}^{s}\left(P_{c_{2}}\right)$ does so below $W_{c_{1}}^{s}\left(P_{c_{1}}\right)$.

Now we proceed to analyse the dynamics given by (5), firstly focusing on the existence of the saddle-saddle heteroclinic trajectory connecting $P_{1}$ with $P_{c}$.

\section{Shooting arguments and the existence of the saddle-saddle connection}

Here we use some shooting arguments to prove the existence of the saddle-saddle heteroclinic trajectory of (5) for a suitable value, $c^{*}>0$, of the speed $c$.

We begin by constructing the set $\mathscr{S}=A A^{\prime} \cup A^{\prime} B^{\prime} \cup B^{\prime} B$, where $A$ and $B$ are the exit (in reverse time) points of $W_{c}^{s}\left(P_{c}\right)$ from $\mathscr{R}$ for $c=c_{0}$ and $c=M^{\frac{1}{2}}$, 


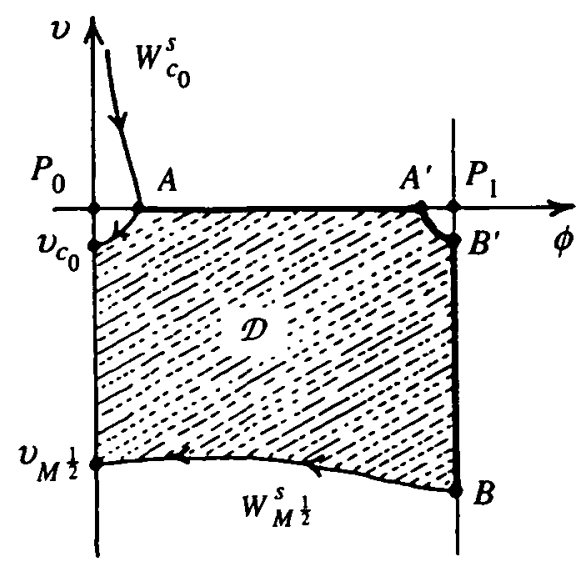

FIG. 3. Construction of the region $\mathscr{D}$.

respectively, and the arc $A^{\prime} B^{\prime}$ is the boundary of a small circular neighbourhood of $P_{1}$ belonging to the region $\mathscr{R}$. The set $\mathscr{S}$ will be useful for shooting (see Fig. 3).

The following lemma holds.

Lemma 2 The stable manifold $W_{c}^{s}\left(P_{c}\right)$, for $c \in\left[c_{0}, M^{\frac{1}{2}}\right]$, has a monotone, continuous intersection with the set $A A^{\prime} B^{\prime} B$.

From Lemma 2 we have the following corollary.

Corollary 1 There exist values $c_{1}<c_{2}$ such that, for $c_{1}<c<c_{2}, W_{c}^{s}\left(P_{c}\right)$ intersects the arc $A^{\prime} B^{\prime}$ monotonically and continuously (and similarly for the subarc $A^{\prime} B^{\prime \prime}$ ).

For the proof of Lemma 2 we need to address one continuity result and to prove a number of preliminary results. The fundamental continuity result we need can be found in many dynamics texts, for example in [22]. This result simply says that, for a hyperbolic equilibrium point, the local stable manifolds not only exist, but they are also continuously dependent on the vector field, in the $C^{r}$ topology $(r \geqslant 1)$. In particular, if the vector field depends smoothly on a parameter (in our case $c$ ) then the stable manifold depends smoothly (and hence continuously) on that parameter.

Let $W_{c, \text { loc }}^{s}\left(P_{c}\right)$ denote the local stable manifold of $P_{c}$. Now define the region $\mathscr{D}$ as the shaded area in Fig. 3.

The following proposition holds:

PROPOSITION $3 \mathscr{D}=\cup_{c_{0} \leqslant c \leqslant M^{\frac{1}{2}}}\left\{W_{c}^{s}\left(P_{c}\right) \cap \mathscr{R}\right\}$ and, furthermore, for each point $(\phi, v) \in \mathscr{D}$, there is a unique $c \in\left[c_{0}, M^{\frac{1}{2}}\right]$ such that $(\phi, v) \in W_{c}^{s}\left(P_{c}\right)$.

Proof. First note that, since $\mathscr{D}$ is compact, every local stable manifold $W_{c, \text { loc }}^{s}\left(P_{c}\right)$ can be extended to a global stable manifold in $\mathscr{D}$, denoted by $W_{c}^{s}\left(P_{c}\right)$. To complete the proof we prove the following proposition.

Proposition 4 For $c \in\left[c_{0}, M^{\frac{1}{2}}\right], W_{c}^{s}\left(P_{c}\right)$ intersects $A A^{\prime} B^{\prime} B$ transversally.

Proof. We know that the $\phi$-coordinate of each $W_{c}^{s}$ is monotonically increasing 
(in negative time); that is, the path of $W_{c}^{s}$ can be written as the graph of a function $v=W_{c}^{s}(\phi)$. Note that we are slightly abusing the notation, since we retain the symbol $W_{c}^{s}$ to denote the graph and the stable manifold of $P_{c}$.

Now it is clear that the right end-point of such a graph must lie on the line $A A^{\prime} B^{\prime} B$. To verify transversality on $A A^{\prime}$ and on $B^{\prime} B$ is an easy matter, since $\dot{v}<0$ on $A A^{\prime}$ and $\dot{\phi}<0$ on $B^{\prime} B$.

To check transversality on the open arc $A^{\prime} B^{\prime}$ we use the outward normal $(\phi-1, v)^{\top}$ to the arc

$$
A^{\prime} B^{\prime}=\left\{(\phi, v) \in \mathscr{R}_{0}:(\phi-1)^{2}+v^{2}=\varepsilon^{2}\right\} \quad(\varepsilon>0 \text { small }) .
$$

We have

$$
\begin{aligned}
(\phi-1, v) \cdot\left(D(\phi) v,-c v-D^{\prime}(\phi) v^{2}-g(\phi)\right)^{\top} \\
\quad=(\phi-1) D(\phi) v+v\left[-c v-D^{\prime}(\phi) v^{2}-g(\phi)\right]<0
\end{aligned}
$$

for $(1-\varepsilon)<\phi<1, v<0,(\phi, v) \in A^{\prime} B^{\prime}$. Thus the flow, in particular $W_{c}^{s}$, is transversal to $A^{\prime} B^{\prime}$.

Now we can finish the proof of Proposition 3. For uniqueness, suppose $(\phi, v)$ lies both on $W_{c}^{s}$ and on $W_{c}^{s}$, for $c \neq c^{\prime}$, say $c<c^{\prime}$. But we know that $W_{c^{\prime}}^{s}<W_{c}^{s}$ as graphs, this implies a contradiction. For existence, we need only translate the continuous dependence of $W_{c}^{s}$ on $c$ into the continuous dependence of the intersection with $A A^{\prime} B^{\prime} B$. To do this, one way is to use what we call Lyapunov surfaces (or complete transverse sections or Poincaré sections): First let $l_{\eta}=$ $\{(\phi, v) \in \mathscr{D}: \phi=\eta\}$ for $\eta>0$ and small ( $\eta$ to the left of $A$; see Fig. 4$)$. We can prove the following claim.

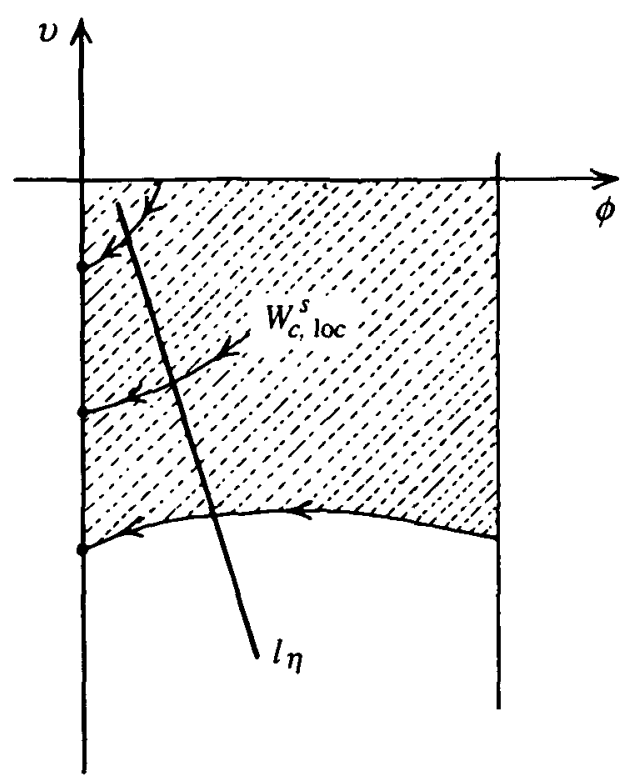

FIG. 4. Intersection of $W_{c}^{s}$ and $l_{\eta}$. 
Claim Every $W_{c}^{s}$ intersects $l_{\eta}$ at a unique point. Let $v_{c}$ be the point of intersection; that is, $v_{c}=W_{c}^{s} \cap l_{\eta}$. Then $v_{c}$ is a continuous, monotone function of $c \in\left[c_{0}, M^{\frac{1}{2}}\right]$.

Proof. Every $W_{c}^{s}$ is transverse to the $v$-axis (since the eigenspace $E_{c}^{s}$ is transverse). Thus, by openness of transverse intersections, $l_{\eta}$ is transverse to $W_{c}^{s}$ Continuous dependence of $W_{c}^{s}$ on $c$ means that the graphs $W_{c}^{s}(\phi)$ depend continuously on $c$ and so, since $v_{c}=W_{c}^{s}(\eta), v_{c}$ is continuous and is uniquely defined. It is monotone because $W_{c^{\prime}}^{s}(\phi)<W_{c}^{s}(\phi)$ for $c<c^{\prime}$, as we know.

We use the term complete transwerse section in $\mathscr{D}$, to denote a segment, such as $l_{\eta}$, transverse to every $W_{c}^{s}, c \in\left[c_{0}, M^{\frac{1}{2}}\right]$ (and in particular intersecting all $W_{c}^{s}$ ).

Define a 'flow' in $\mathscr{D}$ as follows: $\Psi\left(v_{c}, t\right)=\Phi_{c}\left(\left(\eta, v_{c}\right), t\right), v_{c} \in l_{\eta}, t \in \mathbb{R}$, where $\Phi_{c}$ is the flow

$$
\Phi_{c}^{\tau}:\left[P_{M^{1}}, P_{c_{0}}\right] \times(-\infty, 0] \rightarrow \mathscr{D} \subset \mathbb{R}^{2}
$$

such that,

(i) $\Phi_{c}^{0}\left(0,-c / D^{\prime}(0)\right)=P_{c}$,

(ii) $\Phi_{c}^{\tau}\left(0,-c / D^{\prime}(0)\right)=(\phi(\tau), v(\tau)) \in W_{c}^{s}\left(P_{c}\right)$.

The map $\Psi$ uses the stable manifold $W_{c}^{s}$ to propagate the complete transverse section $l_{\eta}$. By the compactness of $\mathscr{D}$, and since every $W_{c}^{s}$ exits $\mathscr{D}$ in negative time, we have $\cup \Psi\left(v_{c}, t\right) \cap \mathscr{D}=\mathscr{D}$, where the union is taken on $t \in \mathbb{R}$ and on $v_{c} \in l_{\eta}$. But $\cup \Psi\left(v_{c}, t\right) \cap \mathscr{D}=W_{c}^{s} \cap \mathscr{D}$. Thus we get Proposition 3 .

To complete the proof of Lemma 2, we need the following key proposition.

Propostrion $5 A A^{\prime} B^{\prime} B$ is a piecewise smooth complete transverse section.

Proof. We have seen that $A A^{\prime} B^{\prime} B$ is transverse to every $W_{c}^{s}$ and that every $W_{c}^{s}$ intersects it.

We now have the correct setting to apply the long-flow-box result to get a map from $l_{\eta}$ to $A A^{\prime} B^{\prime} B$ (see [20]). A long flow box is nothing but a concatenation of local flow boxes.

Let $x_{c}$ be the intersection of $A A^{\prime} B^{\prime} B$ and $W_{c}^{s}$ Parametrize the curve $A A^{\prime} B^{\prime} B$ by using some map $\gamma:[0,1] \rightarrow A A^{\prime} B^{\prime} B$ so that $\gamma(0)=A, \gamma\left(\frac{1}{3}\right)=A^{\prime}, \gamma\left(\frac{2}{3}\right)=B^{\prime}$, and $\gamma(1)=B$, where $\gamma$ is a homeomorphism.

For $x_{1}, x_{2} \in A A^{\prime} B^{\prime} B$, we shall say $x_{1}<x_{2}$ if $x_{1}=\gamma\left(t_{1}\right), x_{2}=\gamma\left(t_{2}\right)$, and $t_{1}<t_{2}$. This allows us to talk about monotone intersections.

In order to conclude the proof of Lemma 2 we need one final proposition.

Proposition $6 x_{c}$ is a continuous, monotone function of $c \in\left[c_{0}, M^{\frac{1}{2}}\right]$.

Proof. Use, for every $v_{c} \in l_{\eta}$, a long flow box to map to $A A^{\prime} B^{\prime} B$ (a homeomorphism can always be found between two complete transverse sections). 


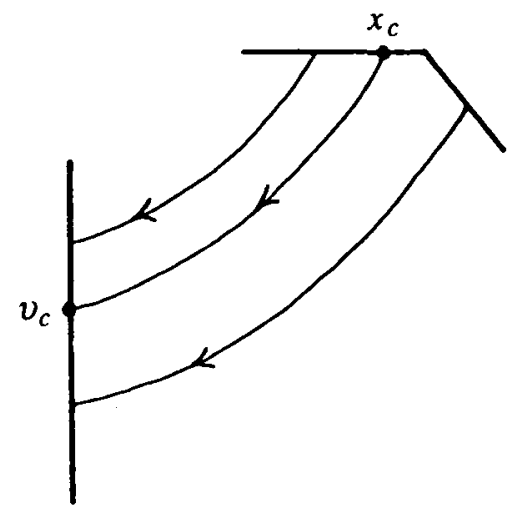

FIG. 5. Proof of Proposition 6. See text for details.

Continuity follows from the continuity of $v_{c}$ and monotonicity similarly follows (see Fig. 5).

With this we have proved Lemma 2.

Now, let us go back to Corollary 1 . By using our parametrization $\gamma$ of $A A^{\prime} B^{\prime} B$, we have a map from $\left[c_{0}, M^{\frac{1}{2}}\right]$ to $[0,1]$ which is a homeomorphism and is such that $c_{0}$ is mapped to 0 and $M^{\frac{1}{2}}$ is mapped to 1 (see Fig. 6).

By the monotonicity, we thus have values $c_{0}<c_{1}<c_{2}<M^{\frac{1}{2}}$ such that $\left[c_{1}, c_{2}\right]$ is mapped $1: 1$ and onto the closed interval $\left[\frac{1}{3}, \frac{2}{3}\right]$, in other words (using $\gamma$ ) to $A^{\prime} B^{\prime}$. This proves Corollary 1.

\section{Uniqueness of the saddle-saddle heteroclinic trajectory}

Finally, to prove that there is a unique value $c^{*} \in\left(c_{0}, M\right)$ such that $W_{c^{*}}^{s}$ connects $P_{c^{*}}$ to $P_{1}$, we note that this reduces to the elementary question of the existence of a fixed point of a continuous map from an interval to itself: $x_{c}$ establishes a homeomorphism between $\left[c_{1}, c_{2}\right]$ and $\left[\frac{1}{3}, \frac{2}{3}\right], x_{c}:\left[c_{1}, c_{2}\right] \rightarrow\left[\frac{1}{3}, \frac{2}{3}\right] \approx A^{\prime} B^{\prime}$. Now we

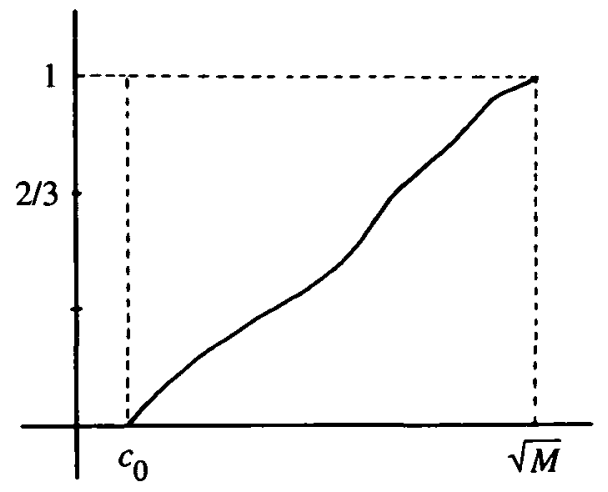

FIG. 6. The map from $\left[c_{0}, M^{\frac{1}{2}}\right]$ to $[0,1]$. 
use the fact that the unstable manifold of $P_{1}$, for $c \in\left[c_{1}, c_{2}\right]$, intersects $A^{\prime} B^{\prime}$. Call the point of intersection $x_{c}^{\prime}$. This is also monotone, but in the opposite direction. Thus we have overall a map from the interval $\left[\frac{1}{3}, \frac{2}{3}\right]$ to itself that is monotone decreasing

$$
\left[\frac{1}{3}, \frac{2}{3}\right] \stackrel{x_{c}^{\prime}}{\longleftarrow}\left[c_{1}, c_{2}\right] \stackrel{x_{c}}{\longrightarrow}\left[\frac{1}{3}, \frac{2}{3}\right]
$$

Thus there must be a fixed point, that is, a $t \in\left[\frac{1}{3}, \frac{2}{3}\right]$, such that $x_{c}(t)=x_{c}^{\prime}(t)$. This is based on the fact that any map of a compact, connected interval into itself has a fixed point (this is the simplest version of the classical fixed-point theorem). Since our map is monotone, the fixed point is unique.

We have thus given an alternative proof to that given in [23] of the uniqueness of a value of $c, c^{*}$, such that $W_{c^{*}}^{s}\left(P_{c^{*}}\right)$ connects $P_{1}$ and $P_{c^{*}}$.

Associated with the $P_{1}-P_{c^{*}}$ heteroclinic saddle connection, whose existence and uniqueness we have just proved, we have the unique travelling-wave solution of sharp type for equation (1). This is because the trajectory $W_{c^{*}}^{u}\left(P_{1}\right)$, of (5), leaving the point $P_{1}$ through the unstable manifold approaches $P_{c^{*}}$ on the negative vertical $v$-axis as time increases. This means that $\phi(-\infty)=1, \dot{\phi}(\tau) \rightarrow-c^{*} / D^{\prime}(0)$ with $v(\tau)=\dot{\phi}(\tau)<0$, and $0 \leqslant \phi(\tau) \leqslant 1$, for all $\tau$ (see Definition 1 ).

If we compare the method carried out here with that given in [23] we see that the method used here involves a more direct dynamical analysis. Hence, the method developed in this paper to prove the existence and uniqueness of the saddle-saddle trajectory of (5) appears to be sufficiently powerful to be applicable in proofs of similar results on heteroclinic connections for other parameterdependent ODE systems, particularly those which come from looking for travelling-wave solutions of parabolic degenerate equations.

\section{Acknowledgements}

FSG wishes to thank the Consejo Nacional de Ciencia y Tecnología (CONACYT, México) and the Facultad de Ciencias of the Universidad Nacional Autónoma de México (UNAM) for their support during the time this research was carried out. $\mathrm{He}$ also thanks the Centre for Mathematical Biology at Oxford for their hospitality. Part of this work was carried out while PKM was visiting the School of Mathematics and Statistics of the University of Sydney.

\section{REFERENCES}

1. Aronson, D. G., \& Weingerger, H. F., 1975. Nonlinear diffusion in population, genetics and nerve pulse propagation. In: Partial Differential Equations and related topics (J. A. Goldstein, ed.). Lecture Notes in Mathematics, No. 446, pp. 5-49.

2. Aronson, D. G., \& Weinberger, H. F., 1978. Multidimensional nonlinear diffusion arising in population genetics. Adv. Maths. 30, 33-76.

3. Aronson, D. G., 1980. Density-dependent interaction-diffusion systems. In: Dynamics and Modelling of Reactive Systems (W. E. Steward, W. H. Ray, \& C. C. Conley, eds.), pp. 161-76. Academic Press.

4. Aronson, D. G., 1985. The role of diffusion in mathematical population biology: 
Skellam revisited, In: Lecture Notes in Biomathematics 57, (V. Cappaso, E. Grosso, \& S. L. Noveri Fontana, eds.). Springer Verlag.

5. Britton, N., 1986. Reaction-Diffusion Equations and their Applications to Biology. New York: Academic Press.

6. Dunbar, S. R., 1984. Travelling wave solutions of diffusive Lotka-Volterra equations: A heteroclinic connection in $\mathbb{R}^{4}$, Trans. Am. Math. Soc. 2, 557-94.

7. ENGLeR, H., 1985. Relations between travelling wave solutions of quasilinear parabolic equations. Proc. Am. Soc. 93, 297-302.

8. FIFE, P. C., \& McLeod, J. B., 1977. The approach of solutions of nonlinear diffusion equations to travelling front solutions, Arch. Rat. Mech. Anal. 65, 335-61.

9. FIFE, P. C., 1979. Mathematical Aspects of Reacting Diffusing Systems. New York: Springer Verlag.

10. GRAY, P., \& ScotT, S. K., 1994. Chemical Oscillations and Instabilities. The International Series of Monographs on Chemistry. Clarendon Press.

11. Grindrod, P., \& Sleeman, B., 1987. Weak travelling fronts for population models with density dependent dispersion. Math. Meth. Appl. Sci. 9, 576-86.

12. Hadeler, K. P., \& Rothe, F., 1975. Travelling fronts in nonlinear diffusion equations. J. Math. Biol. 2, 251-63.

13. HADELER, K. P., 1981. Travelling fronts and free boundary value problems. In: Numerical Treatment of Free Boundary Value Problems (J. Albretch, L. Collatz, \& H. K. Hoffman, eds.). Birkhauser-Verlag.

14. Kappos, E., Hutson, V. C. L., \& Machaikow, K., 1992. Conley index approach to the existence of travelling wave solutions for a scalar degenerate reaction-diffusion equation. Unpublished manuscript.

15. Kolmogorov, A., Petrovsky, I., \& Piskounov, N., 1982. Study of the diffusion equation with growth of the quantity of matter and its applications to biological problems. In: Applicable Mathematics to Non-physical Phenomena (F. Oliveira-Pinto \& B. W. Conolly, eds.). Ellis Horwood Series. Mathematics and Its Applications, John Wiley \& Sons, New York.

16. Kopell, N., \& Howard, L. N., 1975. Bifurcation and trajectories joining critical points. Adv. Maths. 18, 306-58.

17. Murray, J. D., 1989. Mathematical Biology. Biomathematics Texts 19. Springer Verlag.

18. Okuвo, A., 1980. Diffusion and Ecological Problems: Mathematical Models. Berlin: Springer Verlag.

19. Oкuво, A., 1986. Dynamical aspects of animal grouping: Swarms, schools, flocks and herds. Adv. Biophys. 22, 1-94.

20. Palis, J., \& de Melo, W. 1982. Geometrical Theory of Dynamical Systems. Springer Verlag.

21. Perko, L. M., 1993. Rotated vector fields. J. Diff. Eqns. 103, 127-45.

22. Ruelle, D., 1989. Elements of Differentiable Dynamics. Academic Press.

23. Sánchez-Garduño, F., \& Maini, P. K., 1994. The existence and uniqueness of a sharp travelling wave solution for a generalized Fisher-KPP equation. J. Math. Biol. 33, 163-92.

24. SMOLLER, J., 1993. Shock Waves in Reaction-Diffusion Equations. Berlin: Springer.

25. Swinney, H. L., \& KRINSky, V. I. (eds.), 1992. Waves and Patterns in Chemical and Biological Media. Elsevier Science.

26. Winfree, A. T., 1987. When Time Breaks Down. Princeton, N.J.: Princeton University Press. 
